\title{
UM ESTUDO SECCIONAL DE PREVALÊNCIA DE DIABETES TIPO II EM IDOSOS, NO CENTRO DE CONVIVÊNCIA PARA IDOSOS, EM CUIABÁ - MT, EM 2003
}

\author{
Roberto Gomes Azevedo* \\ Miguel Angel Claros Paz** \\ Ivo Antonio Vieira**
}

\section{Resumo}

Esse estudo teve como objetivo geral verificar a prevalência do Diabetes Mellitus tipo II em idosos atendidos no Centro de Convivência para Idosos Padre Firmo na cidade de Cuiabá estado do Mato Grosso, no mês de outubro de 2003, foram entrevistados 600 idosos. Especificamente procurou-se averiguar o motivo da consulta, a freqüência relativa quanto ao sexo/gênero, a freqüência relativa quanto a prática habitual de atividade física, a prática de alimentação dietética e as medidas preventivas adotadas pelos idosos como fatores associados a presença de diabetes tipo II. O trabalho possui uma relevância social onde poderá possibilitar a reorganização de projetos de assistência multiprofissional. Foi um estudo de levantamento quantitativo, transversal, observacional e descritivo, de prevalência, no qual foram utilizados o raciocínio indutivo e a freqüência relativa como procedimento estatístico na apresentação e discussão dos resultados. A população amostral foi constituída por 600 idosos que freqüentaram a instituição no mês de outubro de 2003. Os dados foram apresentados através de tabelas simples. $\mathrm{Na}$ discussão se associou os achados do estudo com a literatura internacional, analisando cada um dos itens. Concluiu-se que a prevalência do diabetes tipo II da amostra estudada $(22,6 \%)$ está acima da prevalência nacional $(17,4 \%)$ e internacional (10\%), atinge principalmente as mulheres idosas. A população amostral não pratica exercícios físicos de forma rotineira $(89 \%)$ e não fazem alimentação dietética $(88 \%)$, mas conhecem de alguma forma as medidas preventivas necessárias para

* Médico Pós-Graduado em Geriatria e Gerontologia, Especialista em Cirurgia Geral pela Associação Médica Brasileira (AMB). E-mail: robertoga @terra.com.br

** Médico Pós-Graduando em Cardiologia; Pós-Graduado em Ginecologia e Obstetrícia; Especialização em Saúde Pública e Pós-Graduado em Geriatria.

*** Médico, Doutor em Saúde e Ambiente, Professor de Biodireito no Centro Universitário Cândido Rondon (UNIRONDON), Pós-Graduação em Perícias Judiciais e Especialista em Urologia e Terapia Intensiva. 
auxiliar no tratamento da doença (61\%).

Palavras-Chave: Idosos. Diabetes Tipo II. Fatores de Risco.

\section{Introdução}

O termo Diabetes Mellitus define um grupo de enfermidades metabólicas. A característica fundamental em todas as formas de manifestação do agravo é a presença de hiperglicemia (aumento dos níveis de glicose no sangue). Isso ocorre como resultado de defeitos na secreção de insulina, em sua ação ou ambos. Essa doença é uma complexa doença na qual ocorre uma alteração generalizada do metabolismo dos carboidratos, lipídios e proteínas. É uma patologia multifatorial onde estão implicados múltiplos fatores em sua patogênese. A prevalência estimada na população adulta é de 7,4\% (1995), com um valor esperado ao redor de $9 \%$ para 2025. (POWERS, 2002, p. 337).

Classifica-se o Diabetes Mellitus segundo o Comitê de Especialistas da Associação Americana de Diabetes (AMERICAN DIABETES ASSOCIATION, 1997) em quatro tipos:

a) Diabetes Mellitus tipo 1;

b) Diabetes Mellitus tipo 2;

c) outros tipos de Diabetes Mellitus;

d) Diabetes Gestacional.

\subsection{Diabetes Mellitus Tipo 1}

No Diabetes Mellitus tipo 1 (não se deve usar o termo Diabetes Insulino dependente), o qual manifesta-se inicialmente na infância ou adolescência. A característica principal é o déficit de insulina, devido à destruição das células beta do pâncreas por processos auto-imunes ou idiopáticos. Dentre os portadores de diabetes a prevalência é de [um] 1 caso em 20 pessoas diabéticas. Nestes casos o corpo produz pouca ou nenhuma insulina. Preco- 
cemente inicia-se o tratamento com injeções diárias de insulina. $\mathrm{O}$ controle mais importante está na dieta e consiste em evitar carboidratos simples (refrigerantes, doces, [etc.]) e não deve sofrer grandes variações na quantidade de calorias diárias. Em não ocorrendo este controle o portador poderá ter surtos de hipoglicemia ou hiperglicemia, que exigem tratamento imediato e urgente sob pena de desenvolver seqüelas ou morte. Os portadores podem desenvolver insuficiência renal antes dos 50 anos. Algumas apresentam insuficiência renal antes dos 30. (POWERS, 2002, p. 337).

\subsection{Diabetes Mellitus Tipo 2}

[ . . . ] não se deve usar o termo Diabetes não Insulino-dependente), o mecanismo fisiopatológico é complexo e não completamente conhecido. Pode ocorrer resistência à insulina por diminuição na resposta dos receptores periféricos. As células beta do pâncreas sofrem exaustão pela constante necessidade de aumento da produção de insulina, motivada pela resistência a insulina. O surgimento é na idade adulta, tendendo a terceira idade associada a obesidade, sedentarismo e maus hábitos alimentares. Pode também ser desencadeada por produtos farmacológicos, como o uso prolongado de corticóides e está freqüentemente associada à hemocromatose não tratada. (POWERS, 2002, p. 337).

\subsection{Diabetes Mellitus Tipo 3}

Estão listados outros tipos de diabetes sendo responsável por menos de $5 \%$ de todos os casos diagnosticados, classificados:

Tipo 3A: defeito genético nas células beta.

Tipo 3B: resistência à insulina determinada geneticamente.

Tipo 3C: doenças no pâncreas.

Tipo 3D: causada por defeitos hormonais.

Tipo 3E: causada por compostos químicos ou fármacos. 


\title{
1.4 Diabetes Gestacional
}

"Ocorre em torno de 2-5\% de todas as gestações. Entre 20 e $50 \%$ destas mulheres desenvolvem diabetes tipo 2 após o término da gravidez." (POWERS, 2002, p. 337).

\begin{abstract}
A Diabetes Mellitus tipo II possui várias características que a distinguem do diabetes tipo I. Ao contrário do diabetes tipos I, há quase sempre alguma produção endógena de insulina nas pessoas com diabetes tipo II, e em geral, pode ser bem-sucedidas se tratada com modificações na dieta e/ou drogas orais. Os pacientes com diabetes tipo II também sofrem de resistência à insulina (seus corpos têm dificuldade em usar a insulina que produzem). Esta doença ocorre tipicamente entre pessoas com mais de 40 anos, em contraste com diabetes tipo I, sendo mais comum entre os obesos. (TIERNEY JÚNIOR.; MCPHEE; PAPADAKIS, 2001, p. 1118).
\end{abstract}

Os dois fatores de risco mais importantes para o diabetes tipo II são uma história familiar positiva e a obesidade. Esta última por aumentar a resistência à insulina. Estudos feitos tanto em homens quanto em mulheres, mostraram que os exercícios regulares podem diminuir substancialmente o risco de se desenvolver diabetes tipo II, mesmo entre pessoas com uma história familiar da doença. Isto em parte é porque os exercícios diminuem a obesidade. Entretanto, mesmo na ausência de perda de peso, o exercício aumenta a sensibilidade à insulina e melhora a tolerância à glicose. (POWERS, 2002, p. 337).

O Censo Nacional de Diabetes de 1988, o último feito pelo Ministério de Saúde descobriu que quase $8 \%$ da população brasileira apresentava a doença diabetes, na faixa de 30 e 69 anos. Em 1980 existia cinco milhões de diabéticos no país, a maioria deles, 9,7\%, em São Paulo e 6,4\% em Recife, onde teve o menor índice. Os dados servem de alerta, pois não é uma enfermidade simples, é a quinta principal causa de morte por doenças e pode causar inú- 
meras complicações graves, como cegueira (mais freqüente entre pessoas de 20 a 74 anos), infarto no miocárdio, derrame cerebral e impotência, além de provocar o risco da amputação de membros. Estima-se que metade das pessoas que desenvolvem a diabetes (pouca mais de dois milhões de pessoas) nem sabem que têm a doença. A prevalência de diabetes na população brasileira é de $7,4 \%$, entretanto na população com faixa etária entre 60-69 anos é de 17,4\%. (BRASIL, 1988, p. 18).

No Centro de Convivência para Idosos Padre Firmo, localizado na cidade de Cuiabá no Estado de Mato Grosso, tem se observado uma alta procura por medicação hipoglicemiante o que motivou o presente estudo quanto à necessidade de determinar a prevalência do diabetes II nesta população no período de trinta dias. Tanto quanto nesse centro o problema ocorre em qualquer unidade recreativa ou de assistência aos idosos.

A importância do estudo está em dar uma visão do quadro de prevalência da doença num determinado ponto da cidade, que embora não possa ser universalizado, pode-se inferir que a quantidade de portadores do agravo é maior do que estes dados possam mostrar. Hoje o diabetes tipo II é um problema de saúde pública e como tal deve ser tratado.

O objetivo do trabalho é mostrar a prevalência do diabetes tipo II entre os idosos do Centro de Convivência Padre Firmo, Cuiabá, MT, através de um estudo seccional realizado no mês de outubro de 2003, analisando a freqüência relativa quanto ao motivo da consulta, sexo, prática de atividade física, uso de alimentação dietética e a adoção de medidas preventivas para os agravos da doença.

\section{Material e Método}

Este é um estudo epidemiológico seccional, avaliados no mês de outubro de 2003, ocorrido no Centro de Convivência para Idosos Padre Firmo, em que cada indivíduo entra na amostra uma única vez. Foram considerados todos os casos que procuraram o Centro de Convivência, 
num total de 600 idosos que atenderam os critérios protocolares. Os critérios para definição da população amostral foi geográfico e políticoadministrativo. Os dados foram coletados através de um questionário orientado abrangendo todas as variáveis deste estudo. Os dados foram apresentados através de tabelas do tipo $2 \times 2$. O tratamento estatístico foi realizado através da análise da freqüência relativa. A discussão foi baseada na literatura e na reflexão sobre os achados.

\subsection{Definição de Termos}

O Diabetes Mellitus é uma síndrome decorrente da falta de produção da insulina; da diminuição da produção de insulina e/ou da incapacidade da insulina produzida exercer adequadamente suas ações. A hiperglicemia (excesso de açúcar no sangue) é o parâmetro para fazer o diagnóstico e avaliar a terapia. (TIERNEY JÚNIOR; MCPHEE; PAPADAKIS, 2001, p. 1118).

\subsubsection{Tipo 1}

Resulta da destruição das células beta pancreáticas, por mecanismo mediado por células, ou seja, através de um processo auto-imune, encerrando a produção de insulina, sendo então as aplicações de insulina a única forma de tratamento. A evolução clínica é rápida; o pico de incidência ocorre na infância e na adolescência, no restante dos portadores o início poderá acontecer em qualquer idade. Algumas formas de diabetes tipo 1 são idiopáticas, ou seja, de origem indeterminada.

\subsubsection{Tipo 2}

Nesse tipo de diabetes, há uma resistência à ação da insulina, em uma combinação com uma deficiência relativa de secreção de insulina. Pessoas classificadas inicialmente com este tipo de diabetes não precisam de insulina para alcançar um controle satisfatório. (TIERNEY JÚNIOR; MCPHEE; PAPADAKIS, 2001, p. 1118). 


\subsubsection{Gestacional}

Este tipo de diabetes é diagnosticado durante a gestação. Há uma resistência insulínica provocada por hormônios produzidos na placenta (prolactina, glucagon, progesterona, lactogênico, placentário), portanto a glicose (açúcar) permanece elevada no sangue materno, facilitando seu transporte e difusão pela placenta até o feto. Os riscos são maiores na mulher que já apresentou diabetes em outras gestações, histórico familiar de diabetes, história de aborto espontâneo, morte intra-uterina ou neonatal, bebes que nasceram com mais de $4 \mathrm{~kg}$ e de má formação congênita. Quanto mais fatores de risco a mulher apresentar, maiores serão as probabilidades de desenvolver diabetes gestacional. (TIERNEY JÚNIOR; MCPHEE; PAPADAKIS, 2001, p. 1118).

\subsection{Metodologia de Inclusão Amostral}

Na fase de pré- inclusão do paciente: o consentimento é obtido por ocasião da consulta, tendo o diagnóstico prévio de Diabetes Mellitus tipo II, e vem em uso de medicamentos hipoglicemiantes.

No procedimento de atendimento médico: o idoso é selecionado de acordo com a sua informação de ser ou não portador da doença.

Variáveis: motivo da consulta, sexo/gênero, praticantes de atividade física, alimentação dietética, medidas preventivas para o controle do diabetes quando da procura do Centro de Convivências para Idosos.

Critério de idoso: pacientes com mais de 60 anos e cadastrados no Centro de Convivências para Idosos.

Critérios de inclusão: considerou-se portador Diabetes Mellitus tipo II todo paciente com glicemia acima $110 \mathrm{mg} / \mathrm{dl}$, e foram incluídos todos os pacientes com mais de 60 anos de idade que procuraram o Centro de Convivência para Idosos de Cuiabá - MT no mês de outubro de 2003; foram incluídos os novos pacientes que procuraram a instituição pela primeira vez e aqueles em tratamento crônico. 
O critério do Diabetes Expert Commitee na avaliação do teste padrão de tolerância à glicose oral, uma glicemia maior que $126 \mathrm{mg} / \mathrm{dl}$ é diagnosticada como diabetes, levando em consideração que se caso a glicemia for menor que $126 \mathrm{mg} / \mathrm{dl}$, outros critérios devem ser anexados, tais como os pontos entre 0 e $120 \mathrm{mg} / \mathrm{dl}$ e a dosagem duas horas após a sobrecarga de glicose (mg/dl). (TIERNEY JÚNIOR; MCPHEE; PAPADAKIS, 2001, p. 1123).

Estes critérios diagnosticados contidos na tabela estão baseados nas novas recomendações.

TABELA 1 - Parâmetros adotados para a caracterização do Diabetes Mellitus, segundo o Diabetes Expert Commitee

\begin{tabular}{l|r|r}
\hline & \multicolumn{1}{|c|}{ Jejum } & 2 hs após 75g de glicose \\
\hline Tolerância à Glicose & $>110 \mathrm{a}<126$ & $140 \mathrm{a}<200$ \\
\hline Diabetes Mellitus & $>126$ & $>200$ \\
\hline
\end{tabular}

Fonte: American Diabetes Association, 2003.

Critérios de exclusão: aqueles pacientes idosos que não informaram ser portadores de diabetes e com exames de glicemia em jejum abaixo de $110 \mathrm{mg} / \mathrm{dl}$. Todos os pacientes com menos de 60 anos e os com pressão arterial normal.

Não foram realizados exames de tolerância a glicose.

Cálculo estatístico: foi utilizada somente a freqüência relativa como tratamento dos dados coletados.

\section{Resultados}

TABELA 2 - Determinação da freqüência relativa da prevalência de Diabetes Mellitus tipo II, quanto ao motivo da consulta

\begin{tabular}{l|r|r}
\hline \multicolumn{1}{c|}{ Motivo da Consulta } & F & \multicolumn{1}{c}{$\%$} \\
\hline Diabetes & 136 & 22,6 \\
\hline Outros & 464 & 77,4 \\
\hline Total & 600 & 100
\end{tabular}

Fonte: Pacientes atendidos no ambulatório do Centro de Convivência para Idosos, no mês de outubro de 2003. 
A Tabela 2 mostra que $22,6 \%$ dos idosos que procuraram o Centro de Convivência para Idosos, que corresponde a uma fração elevada da população. Segundo Powers (2002, p. 337) o número de pessoas com Diabetes Mellitus aumenta com a idade da população, sendo que a incidência de $1,5 \%$ em indivíduos de 20 a 39 anos, aumentando para $20 \%$ nos indivíduos acima de 75 anos.

TABELA 3 - Determinação da freqüência relativa da prevalência de Diabetes Mellitus tipo II, quanto ao sexo/gênero

\begin{tabular}{l|l|l}
\hline \multicolumn{1}{c|}{ Sexo / Gênero } & \multicolumn{1}{c|}{ F } & \multicolumn{1}{c}{$\%$} \\
\hline Masculino & 55 & 40,45 \\
\hline Feminino & 81 & 59,55 \\
\hline Total & 136 & 100
\end{tabular}

Fonte: Pacientes atendidos no ambulatório do Centro de Convivência para Idosos, no mês de outubro de 2003.

A Tabela 3 evidencia que o maior percentual dos idosos portadores de Diabetes Mellitus tipo II, é de idosos do sexo feminino. Powers (2002, p. 337) afirma que a prevalência da Diabetes Mellitus é similar em homens e mulheres quando se aproximam da idade dos 50 anos. Após os 60 anos é mais freqüente em homens acima de 60 anos. Afirma ainda que as razões para estas diferenças ainda não são claras.

TABELA 4 - Determinação da freqüência relativa da prevalência de Diabetes Mellitus tipo II, quanto a prática de atividade física

\begin{tabular}{l|l|l}
\hline Prática de Atividade Física & \multicolumn{1}{|c|}{ F } & \multicolumn{1}{c}{$\%$} \\
\hline Sim & 66 & 11 \\
\hline Não & 534 & 89 \\
\hline Total & 600 & 100 \\
\hline
\end{tabular}

Fonte: Pacientes atendidos no ambulatório do Centro de Convivência para Idosos, no mês de outubro de 2003.

A Tabela 4 evidencia que o percentual maior é de idosos sedentários (89\%), diabéticos ou não. Acomete $90 \%$ da população com diabetes, dentre eles a maioria são obesos, hipertensos, sedentários e prin- 
cipalmente apresentam história familiar. "Seus sinais e sintomas podem passar sem diagnóstico durante muitos anos, aumentando os riscos de se desenvolver complicações microvasculares e macrovasculares do diabetes." (POWERS, 2002, p. 337).

TABELA 5 - Determinação da freqüência relativa da prevalência de Diabetes Mellitus tipo II, quanto a alimentação dietética

\begin{tabular}{l|l|l}
\hline \multicolumn{1}{c|}{ Alimentação Dietética } & \multicolumn{1}{c|}{ F } & \multicolumn{1}{c}{ \% } \\
\hline Sim & 72 & 12 \\
\hline Não & 528 & 88 \\
\hline Total & 600 & 100 \\
\hline
\end{tabular}

A Tabela 5 mostra que os idosos em estudo não se preocupam com cuidados alimentares como medidas preventivas. Segundo Tierney Júnior, McPhee e Papadakis (2001) uma dieta bem balanceada e nutritiva é um elemento fundamental da terapia. Entretanto mais da metade dos casos, os pacientes diabéticos falham em seguir a dieta.

TABELA 6 - Determinação da freqüência relativa da prevalência de Diabetes Mellitus tipo II, quanto a medidas preventivas

\begin{tabular}{l|l|l}
\hline \multicolumn{1}{c|}{ Medidas Preventivas } & \multicolumn{1}{c|}{ F } & \multicolumn{1}{c}{$\%$} \\
\hline Sim & 366 & 61 \\
\hline Não & 234 & 39 \\
\hline Total & 600 & 100 \\
\hline
\end{tabular}

Fonte: Pacientes atendidos no ambulatório do Centro de Convivência para Idosos, no mês de outubro de 2003.

A Tabela 6 mostra que a maior parte dos idosos do estudo participaram de algum tipo de campanha preventiva. Mais da metade restringe-se a saber que o uso de carboidratos não é recomendado. Sabem também que devem fazer alguma forma de dieta. 


\section{Discussão dos Resultados}

De acordo com a Organização Mundial da Saúde, em 2006 há cerca de 171 milhões de pessoas doentes da Diabetes, e esse índice aumenta rapidamente, é estimado que em 2030 esse número dobre. A Diabetes Mellitus ocorre em todo o mundo, mas é mais comum (especialmente a tipo II) nos paises mais desenvolvidos. O maior aumento atualmente é esperado na Ásia e na África, onde a maiorias dos diabéticos serão vistos em 2030. O aumento do índice de Diabetes em paises em desenvolvimento segue a tendência de urbanização e mudança de estilos de vida (UNITED KINGDOM..., 1998).

A Diabetes está entre as dez mais, se não as cinco mais, doenças de maior índice de morte no mundo, e está aumentando de forma inexorável. Por pelo menos 20 anos, o número de diabéticos na América do Norte está aumentando consideravelmente. Em 2005 eram em torno de 20.8 milhões de pessoas com diabetes somente nos Estados Unidos. De acordo com a American Diabetes Association existem cerca de 6.2 milhões de pessoas não diagnosticadas e cerca de 41 milhões de pessoas que poderiam ser consideradas "pré"-diabéticas. Os Centros de Controles de Doenças classificaram o aumento da doença como epidêmico, e a National Diabetes Information Clearinghouse (NDIC) fez uma estimativa de custo de $\$ 132$ bilhões de dólares, somente para os Estados Unidos este ano (UNITED KINGDOM..., 1998).

Verifica-se que os idosos são pacientes geralmente acometidos por mais de uma patologia, fazem uso de diferentes medicamentos (polimedicados), e realizam vários exames laboratoriais para o diagnóstico e acompanhamento. Considerando-se que tais exames são utilizados para nortear condutas, a interpretação adequada dos resultados, deverá sempre que possível levar em consideração as possíveis interferências dos medicamentos.

A incidência de diabetes se encontra acima de $22 \%$ do total do atendimento ambulatorial e que a referência na literatura é de 17,4\% (Tabela 2). A maioria dos idosos diabéticos é do sexo feminino (Tabela $3)$.

Aproximadamente $10 \%$ das pessoas diabética têm diabetes do tipo 1. Contudo, o número de pessoas com diabetes tipo 2 está aumentando dramaticamente em decorrência de inúmeros fatores, tais como: 
idade da população; aumento das taxas de obesidade; modo de vida com aumento do sedentarismo; aumento da incidência de diabetes do tipo 2 em crianças nascidas em populações de alto risco. (TIERNEY JÚNIOR; MCPHEE; PAPADAKIS, 2001, p. 1125).

A maior parte dos idosos em estudo (89\%) não praticam atividade física (Tabela 4). Powers (2002) afirma que num grande estudo realizado no Canadá, as pessoas com risco de desenvolvimento de diabetes tipo II, podem diminuir o risco em $58 \%$ se exercitando moderadamente por 30 min por dia e perdendo de $5-7 \%$ do peso corporal. Em pessoas acima de 60 anos, o risco pode ser minorado em $71 \%$.

Os idosos em estudo não se preocupam com cuidados alimentares como medidas preventivas (Tabela 5). Tierney Júnior, McPhee e Papadakis (2001) afirmam que a tentativa de redução de peso é um objetivo frustrante, que somente pode ser atingido através da supervisão estrita e da educação do paciente obeso. Tanto a literatura clínica quanto a epidemiológica enfatiza o uso da dieta e do exercício físico regular como medidas essenciais para o controle da glicemia prevenção de complicações, mesmo em vigência de tratamento medicamentoso (ALCOLADO, 1998).

A atividade física, segundo Powers (2002), é muito importante no controle do diabetes. Participar de um programa regular de atividade física pode melhorar os níveis de glicose no sangue das pessoas idosas com diabetes.

Por outro lado, é bem conhecida a dificuldade de adesão a tratamentos que impliquem mudanças de comportamento (GREGERMAN, 1995). Há evidências na literatura de que o maior contato com serviços de saúde tem um efeito protetor sobre a saúde, estando relacionado a uma probabilidade menor de morrer por diabetes (DORMAN, 1985). Uma vez que mais da metade dos pacientes estiveram consultando no serviço nos últimos dois meses, é provável que um número razoável de oportunidades para o desenvolvimento de ações que promovam a adesão a medidas não farmacológicas de controle do diabetes, como a dieta e o exercício físico regular, estejam sendo perdidas.

Geralmente o exercício é negligenciado como tratamento para diabetes. Porém, o exercício melhora o controle da glicemia no sangue, ajuda a circulação, reduz a resistência da insulina e melhora a saúde vascular. $\mathrm{O}$ exercício também mantém o peso, eliminando um fator de 
complicação do diabetes, e aumenta os músculos, os quais processam melhor o açúcar do que os tecidos gordurosos.

A maior parte dos idosos do estudo participaram de algum tipo de campanha preventiva (Tabela 6). O manejo do diabetes tipo II envolve a necessidade de planejar as comidas e comer de forma correta são condutas fundamentais do controle dos níveis de glicose no sangue, a pressão arterial e o colesterol. As metas de controle de peso e da atividade física diária, são fundamentais para o diabético tipo II.

É muito importante, para pessoas com doença crônica, saber que o exercício melhora o humor e aumenta a energia. Praticando exercícios, muitas vezes os pacientes podem reduzir a dose de insulina ou medicamentos orais.

Segundo um estudo editado no Jornal da Associação Médica Americana (CLARKE; COX; GONDER-FREDERICK; KOVATECHEV, 1999), exercícios podem reduzir estes riscos da seguinte maneira:

a) melhorando a função das células endoteliais, as quais protegem os vasos sangüíneos e ajudam a alargarem os vasos para melhorar o fluxo sangüíneo nos períodos de estresse;

b) fortalecendo o ventrículo esquerdo do coração para melhorar a habilidade de bombeamento do mesmo;

c) reduzindo o endurecimento dos vasos sangüíneos, um sinal de doença cardíaca prematura;

d) reduzindo a gordura total e abdominal para melhorar a sensibilidade da insulina e baixar a pressão arterial.

Mas na falta de insulina suficiente, principalmente no caso do diabetes tipo I, o exercício poderá elevar o nível do açúcar no sangue. Quando se passa de um exercício moderado para um vigoroso, ocorre um estímulo no sentido de que o fígado libere glicose armazenada. Se não houver insulina suficiente para enfrentar o açúcar extra no sangue, essa ficará concentrada no sangue, podendo causar hiperglicemia. $\mathrm{O}$ importante é aliar uma dieta saudável com exercícios regulares, sem exageros.

Os dados obtidos deverão ser interpretados de forma restritiva levando-se em consideração as características da população estudada, restrita à uma clientela de idosos no Centro de Convivência para Idosos Padre Firmo em Cuiabá, no mês de outubro de 2003, com demanda 
espontânea e abrangendo diferentes níveis sociais.

\title{
5 Conclusões
}

Os dados evidenciam ser a prevalência de diabetes tipo II entre idosos desta amostra superiores $(22,6 \%)$ a valores encontrados na literatura nacional $(17,4 \%)$ apesar de trabalhos internacionais demonstrarem percentuais mais elevados em determinadas regiões.

O percentual de portadores de diabetes tipo II entre os idosos do estudo, ficou evidente que é mais prevalente entre aqueles do sexo feminino $(59,5 \%)$.

A atividade física é um bom coadjuvante no controle da hiperglicemia e na minimização dos riscos cardiovasculares diminuindo o surgimento da ateroesclerose. $\mathrm{Na}$ amostra o sedentarismo foi um dado significante estatisticamente, uma vez que $89 \%$ dos idosos não praticam atividade física regular.

A falta de uma dieta alimentar adequada, equilibrada ficou evidenciada na amostra uma vez que $88 \%$ dos idosos não praticam uma alimentação dietética.

$\mathrm{O}$ conhecimento da necessidade de praticar alguma forma de medida preventiva no sentido de diminuir os riscos do diabetes tipo II, quer seja através de campanhas de conscientização ou sob a forma de palestras esclarecedoras é um dado importante sendo que $61 \%$ dos portadores do diabetes tipo II informaram ter alguma forma de conhecimento neste sentido.

\section{A SECCIONAL STUDY OF PREDOMINANCE OF DIABETES TYPE II IN ELDER, AT CENTRO DE CONVIVENCIA TO EL- DERS, IN CUIABÁ - MT, IN 2003}

\begin{abstract}
This study had the general objective to check the predominancy of Diabetes Mellitus type II in elder people that had being seen on the living center of elder people Padre Firmo on Cuiabá city, Mato Grosso, in October, 2003, were interviwed 600 elder. Looking specifically to
\end{abstract}


discover the reason of the visiting, the relative frequency habit of doing physical activities, to live on healthy diet and the prevention resource that elderly people used to associate the fact of the presence of diabetes type II. The study has a social importance that can turn possible the reoganization of profissional assistance projetcts. The study was a quantitative survey transversal, observable and discribable of predominace, also had being used the induction reasoning and the relative frequency as statistical proceedings on the relative frequency as statistical proceedings on the presentation and discussion of the results, the data had being introduced throught simple tables. On the discussion had being associated the things discoverde on the study with the international literature, analusing each one of the iten analysed. It follows that the prevalence of diabetes type II of the sample studied $(22,6 \%)$ is above the national prevalence $(17,4 \%)$ and international $(10 \%)$ principally reaching elder women. The sample population composed of 600 elder that had being on the institution on october 2003, didn't have a routine of praticing exercises $(89 \%)$; didn't have a healthy diet $(88 \%)$ but knew some how the necessary prevention resource to help on the disease treatment (61\%).

Keywords: Elder. Diabetes Type II. Risk Fators.

\section{REFERÊNCIAS}

ALCOLADO John. Genetics of Diabetic Complications. Lancet, London, v. 351, p. 230-237, 1998.

AMERICAN DIABETES ASSOCIATION. Clinical Practice Recomendations. Diabetes Care, Alexandria, v. 21, 1997. Supplement 1.

AMERICAN DIABETES ASSOCIATION. Report of the Expert Committee on the Diagnosis and Classification of Diabetes Mellitus. Diabetes Care, Alexandria, v. 26, 2003. Supplement 1.

BRASIL. Ministério da Saúde. Conselho Nacional de Desenvolvimento Científico e Tecnológico. Censo Nacional de Diabetes. Brasília, DF, 1988. 
CLARKE, William L.; COX, Daniel J.; GONDER-FREDERICK, Linda A.; KOVATCHEV, Boris. Hypoglycemia and the Decision to Drive a Motor Vehicle by Persons with Diabetes. JAMA, Chicago, v. 282, p. 750-754, 1999.

DORMAN, Janice S. et al. The Pittsburg Insulin-Dependent Diabetes Mellitus (IDDM) Morbidity and Mortality Study: case-control analyses of risk factors for mortality. Diabetes Care, Alexandria, v. 8, p. 54-60, 1985.

GREGERMAN, Robert I. Diabetes Mellitus. In: BARKER, L. Randol; BURTON, John R.; ZIEVE, Philip D. Principles of Ambulatory Medicine. 4th ed. São Paulo: Hucitec, 1995. P. 977-1019.

POWERS, Alvin C. Diabetes Mellitus. In: FAUCI, Anthony S. et al. (Ed.). Harrison: princípios de Medicina Interna. 15. ed. São Paulo: Guanabara Koogan, 2002. P. 2186-2208.

TIERNEY JÚNIOR, Lawrence M.; McPHEE, Stephen J.; PAPADAKIS, Máxime A. Diagnóstico e Tratamento: um livro médico. São Paulo: Atheneu, 2001 .

UNITED KINGDOM PROSPECTIVE DIABETES STUDY GROUP. Tight Blood Pressure Control and Risk: of macrovascular and microvascular complications in type 2 diabetes: UKPDS 38. British Medical Journal, London, v. 317, n. 7160, p. 703-712, 1998.

Recebido: 20/10/2005 